\section{Efecto fungicida del quitosano sobre la roya inoculada en cafeto}

\section{Fungicidal effect of chitosan on rust inoculated in coffee}

\author{
Mónica Yari Oviedo* Juan Sebastián Morales * \\ Juan Sebastián Ramírez - Navas**
}

* Químico, Programa de Química, Universidad Santiago de Cali. ** Ingeniero Químico, PhD en Ingeniería, Profesor. GIEMA, Universidad Santiago de Cali - GIPAB, Universidad del Valle, C.P. 25360, Cali, Colombia.

\section{Resumen}

En Colombia el cultivo de café es uno de los más importantes del país y se realiza en casi todo el territorio; la especie cultivada es la Coffea arábica con múltiples variedades; entre las más populares y de mejor sabor se encuentra la Caturra, la cual a pesar de poseer las grandes propiedades es atacada por el hongo parásito Hemileia vastatrix, mejor conocido como Roya. En este trabajo se evaluó el efecto del compuesto quitosano sobre el hongo Hemileia vastatrix, con el fin de determinar las propiedades fungicidas de dicho compuesto y poderlo considerar como una alternativa natural para combatir la enfermedad en éstas plantas. Inicialmente, se realizó la obtención del quitosano a partir de la quitina proveniente de cáscara de camarón; mediante un proceso químico se obtuvieron dos clases de quitosano uno con un grado de desacetilación del $28,94 \%$ y otro con un grado de desacetilación del $56,49 \%$, los cuales se compararon con otros dos compuestos de quitosano uno de bajo peso molecular $(62 \mathrm{cps})$ y otro de medio peso molecular (598 cps). Para determinar experimentalmente la efectividad de este compuesto en sus distintas formas químicas, se preparó un germinador el cual fue sembrado con Coffea arábica variedad Caturra y se aplicó a éste un diseño estadístico de bloques al azar; se escogió además como variable a observar durante las fases de la infección con Roya, el número de hojas enfermas en cada planta sembrada. Los compuestos de quitosano se prepararon en ácido clorhídrico a una concentración del $1 \% \mathrm{p} / \mathrm{v}$ y se aplicaron a cada planta por aspersión. Los resultados encontrados mostraron la actividad fungicida del compuesto cuando se aplica como tratamiento preventivo, es decir antes del ataque de la enfermedad a las plantas, y también cuando se aplica a plantas que si tienen Roya en su fase incial.

Palabras clave: Coffea arábica, caturra, fitoprotección, femileia vastatrix, quitina desacetilada.
Recibido: $12 / 04 / 2016$

Revisado: 21/06/2016

Aceptado: 01/11/2016

Correspondencia de autor: juan.sebastian.ramirez@ correounivalle.edu.co

(C) 2016 Universidad La Gran Colombia. Este es un artículo de acceso abierto, distribuido bajo los términos de la licencia Creative Commons Attribution License, que permite el uso ilimitado, distribución y reproducción en cualquier medio, siempre que el autor original y la fuente se acrediten.

Cómo citar:

Oviedo. M., Morales S., Ramírez-Navas. J (2016)Efecto fungicida del quitosano sobre la roya inoculada en cafeto. UGCiencia22, 45-56.

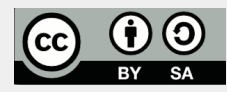




\begin{abstract}
In Colombia, coffee growing is one of the most important crops, and it is carried out almost in the whole territory, the Arabic Coffea with multiple varieties is grown; the most popular and best flavor is Caturra, which, in spite of its great properties, is attacked by parasitoid fungus Hemileia vastatrix, best known as Rust. This project evaluated the effect of chitosan compound on Hemileia vastatrix fungus, in order to establish fungicide properties of such compound, and consider it as a natural alternative to combat such disease of these plants.

At first, chitosan was obtained from shrimp shell Chitin; two kinds of chitosan, one with a deacetylation degree of $28,94 \%$, and other with deacetylation degree of $56,49 \%$, were obtained through a chemical process, which were compared to other two chitosan compounds, one of a low molecular weight (62 cps) and other of molecular middle weight (598 cps). In order to experimentally establish effectiveness of this compound, in its various chemical forms, a germinator was prepared and planted of Coffea Arabic Caturra variety, and a statistical design of random blocks was applied. In addition, the number of diseased leaves of each plant was selected as the variable to be observed during phases infected by Rust. Chitosan compounds were prepared in chloride acid at a $1 \%$ and $\mathrm{p} / \mathrm{v}$ concentration, and applied to each plant by spray. Results showed fungicide activity of the compound when applied as a preventive treatment, that is, previously to be attacked by the disease, and also when applied to Rust infected coffee plants during its initial phase.
\end{abstract}

Keywords: Arabic Coffea, Caturra, phytoprotection, Hemileia vastatrix, deacetylated chitin.

\title{
Introducción
}

La roya (Hemileia vastatrix) es una de las enfermedades más destructivas de los cultivos de café y genera gran impacto a nivel mundial. $\mathrm{Su}$ ataque sobre la planta se manifiesta con necrosis del área afectada y caída de las hojas con posterior pérdida de frutos y disminución de la cosecha (Montes, Armando P, \& Cadena, 2012). El incremento en el uso de agroquímicos para combatir la roya incrementa los costos de producción.

En Colombia, se han presentado dos ataques con pérdidas económicas considerables para los caficultores, el primero ocurrió en el año 1983 y el segundo entre 2008 y 2011. Los daños al cultivo fueron de más del $30 \%$ sin posibilidades de efectuar ningún control químico (Cristancho, Rozo, Escobar, Rivillas \& Gaitán, 2012). A raíz de esta situación se han desarrollado estudios para prevenir y controlar la proliferación de la roya en el café. Dichas investigaciones abarcan desde la adopción de aplicación de fungicidas antes y/o después de la aparición de la enfermedad, hasta la creación de nuevas variedades genéticamente más resistentes. En Colombia, a pesar de las nuevas variedades desarrolladas, son muchos los terrenos en los que se sigue cultivando la variedad Caturra, aproximadamente 350000 hectáreas de las 900 000 que existen en todo el territorio nacional. Las alternativas amigables con el medio ambiente para controlar la roya no han sido prometedoras, se implementó el manejo biológico empleando microorganismos como Bacillus thuringiensis, Pseudomonas fluorescens, P. aureofaciens, P. alcaligenis, P. putida y Lecanicillium lecanii (Rivillas Osorio, Serna Giraldo, Cristancho Ardila \& Gaitán Bustamante, 2011) pero los esfuerzos resultaron infructuosos.

Existen compuestos con poder fungicida que son biodegradables y no contaminantes. El quitosano es uno de estos (Kean \& Thanou, 2010). Es un polímero que se obtiene a partir de la transformación química de la quitina contenida en el exoesqueleto de crustáceos 
(Aider, 2010). Está conformado por unidades de D-glucosamina y N-acetil-D-glucosamina. Su actividad sobre hongos se manifiesta con reducción del crecimiento micelial y germinación del hongo (Younes, Sellimi, Rinaudo, Jellouli \& Nasri, 2014). Este compuesto derivado de la quitina, además de la propiedad mencionada, incrementa los mecanismos de defensa en los vegetales y plantas (Prapagdee, Kotchadat, Kumsopa \& Visarathanonth, 2007; Rodriguez, Ramírez, Cárdenas Rodriguez, \& Bautista Baños, 2006; Van, Minh \& Anh, 2013); generando así un doble efecto en su aplicación (Hadwiger, 2013). El objetivo de este trabajo fue estudiar el efecto del quitosano sobre Hemileia vastatrix que ataca a la especie Coffea arábica.

\section{Materiales y métodos}

\section{Germinación del café}

El germinador se construyó empleando madera para las paredes y guadua para el piso (Figura 1). Las dimensiones del cajón fueron de $100 \mathrm{~cm}$ x $100 \mathrm{~cm}$ x $30 \mathrm{~cm}$. Para la siembra de las plantas se seleccionaron las semillas de variedad Caturra. Se colocó una capa de teja española picada en el fondo del cajón, seguida por una capa de tierra, una capa de arena, una capa tierra y arena mezcladas. Se sembraron las semillas seleccionadas dejando un espacio de $1 \mathrm{~cm}$ entre cada una, y se procedió a humedecer la tierra. Se esperó el brote de plantas. Aquellas plantas cuya raíz se encontraba bien formada, completa y fuerte se trasplantaron a bolsas de $17 \mathrm{~cm} \mathrm{x}$ $20 \mathrm{~cm}$. Antes del trasplante, la bolsa se llenó con tierra y cascarilla de arroz y las plantas se desprendieron del germinador manteniéndolas sobre papel húmedo hasta ser sembradas. En este proceso se verificó que la raíz no sufriera daños mecánicos.

\section{Preparación del inóculo}

Para la preparación del inóculo de roya se siguióla metodología de Rozo-Peña y Cristancho-Ardila (2010). Se procedió a recolectar hojas con presencia de roya de un lote comercial de café ubicado en el sector de La María (Cali, Valle del Cauca). Se llevaron inmediatamente a los laboratorios de la Universidad Santiago de Cali. Se recogieron con una espátula las esporas de color naranja y se preparó una solución de $0,25 \mathrm{~g}$ en $500 \mathrm{~mL}$ de agua. La solución preparada se aplicó con un gotero en cantidad suficiente para cubrir todo el envés de las hojas. Las plantas de seis meses de edad se dejaron en oscuridad durante 48 horas para facilitar el proceso de infección. La aplicación se realizó en las plantas seleccionadas para el método preventivo y para el curativo.

\section{Diseño experimental}

Las unidades experimentales sobre las cuales se aplicaron los tratamientos correspondieron a plantas de café que ya tenían seis meses de edad variedad Caturra y se encontraban en el almácigo.

Antes de iniciar los tratamientos y para reducir el error experimental, se seleccionaron del germinador al azar unidades experimentales homogéneas con edad menor de seis meses (un total de 72 plantas), que cumplieron los siguientes criterios: 1) plantas con crecimiento de $10 \mathrm{~cm}, 2$ ) hojas bien formadas y 3) hojas y tallo sanos. Se realizó una aleatorización con un solo criterio de bloques en donde todos los tratamientos ocurrieron igual número de veces en cada bloque (figura 1). En principio, se delimitaron seis bloques de igual tamaño en el germinador para obtener seis plantas de cada uno y obtener así un total de 36 plantas a las que se les aplicó el método preventivo. Después se extrajeron otras seis de cada bloque para un 
total de 36 plantas, para el método curativo. Estas plantas se extrajeron del germinador conservando la diferencia por bloques en el almácigo.

Figura 1. Selección de unidades experimentales en los bloques en el germinador, con plantas de café de edad menor a seis meses variedad Caturra.

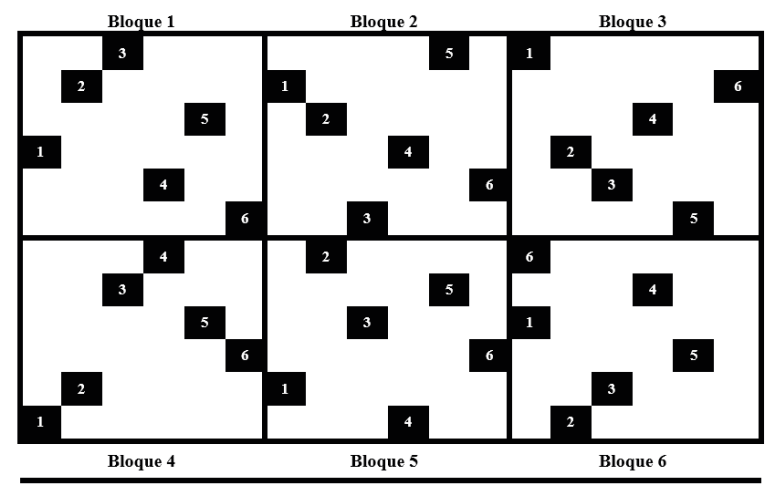

Como factor se tuvieron el compuesto aplicado y el método. Los niveles para el factor compuesto fueron quitosano con bajo grado de desacetilación (QBGD), quitosano con medio grado de desacetilación (QMGD), quitosano con bajo peso molecular (QBPM), quitosano con medio peso molecular (QMPM), solución de $\mathrm{HCl} 0,1 \mathrm{M}$ (ACIDO) y testigo (AGUA). A cada planta se le asignó aleatoriamente un compuesto. Este procedimiento se realizó en dos etapas, primero para el método preventivo y luego para el método curativo. Se realizaron mediciones repetidas de las plantas con cada tratamiento en dos momentos sobre las mismas unidades experimentales al inicio de la infección y al final de la infección.

Se consideraron los factores perturbadores, controlables y no controlables en el experimento, con el fin de valorar su efecto en los resultados y plantear alguna estrategia que permitiera inhibirlo. Así se logró inhibir el efecto perturbador de la agitación y temperatura en la obtención de quitosano, la agitación en la caracterización potenciométrica, la lluvia acumulada y la luz solar en la aplicación de compuestos antes y después de la inoculación con roya. Sin embargo, no se logró inhibir el efecto perturbador de la temperatura, la humedad relativa y el viento en la aplicación de compuestos.

La variable dependiente fue el número de hojas enfermas, y la variable de exposición (offset) se tuvo el logaritmo del número de hojas totales.

\section{Compuestos}

En el laboratorio se obtuvieron dos compuestos de quitosano a partir de quitina según el método de Mohammed et al. (2013), que cumplieron con las características: QBGD $((50 \%, 6 \mathrm{~h}) 28,94 \mathrm{GD})$ y QMGD $((25 \%, 2 \mathrm{~h})$ 56,49 GD). Los compuestos se caracterizaron mediante análisis potenciométrico (Parada et al., 2004; Hidalgo et al., 2008) y análisis IR con transformada de Fourier. Se utilizaron dos compuestos comerciales de quitosano: QBPM (62 cps, 82,6 GD) y QMPM (598 cps, 81 GD - Sigma-Aldrich). También se empleó agua y solución de ácido clorhídrico $0,1 \mathrm{M}$.

\section{Forma de aplicación de los compuestos}

Para la aplicación de los tratamientos se realizó un riego en forma de aspersión de los polímeros preparados en ácido clorhídrico $0,1 \mathrm{M}$; la concentración de la solución empleada de cada tratamiento fue al $1 \% \mathrm{p} / \mathrm{v}$; la dosis colocada sobre cada colino (plantas de seis meses) que estaba en el álmacigo de café fue de $5 \mathrm{~mL}$. Cada método tuvo su forma de aplicación la cual se describe a continuación: 
Aplicación de compuestos para método preventivo. Se aplicaron los compuestos antes de inocular la roya. Se aplicaron dos capas sobre las unidades experimentales. Entre cada aplicación se dejó un espacio de cuatro días.

Aplicación de compuestos para método curativo. Los compuestos de quitosano se aplicaron después de inocular la roya. La aspersión se efectuó en dos capas, la primera al inicio de la infección y la segunda a la mitad del ciclo infeccioso, antes de la producción de esporas.

\section{Análisis estadístico}

Se realizó un análisis descriptivo y un análisis de regresión. En el primero se representó gráficamente el número de hojas enfermas según el compuesto y el método de aplicación, compuestos de quitosano para cada etapa de medición, por bloques y por etapa. También se describió la distribución del número de hojas utilizando como medida de tendencia central la mediana (cuartil 2) y como medida de dispersión el rango del número de hojas. En el segundo se evaluó el modelo de regresión que tiene como variable de respuesta el número de hojas enfermas y variables de predicción del compuesto y método. En este modelo se asumió que hay un efecto del bloque que afecta a la relación entre el número de hojas enfermas y el tratamiento y que hay un efecto de la planta que afecta la relación entre el número de hojas enfermas y el tratamiento. Esto para considerar respectivamente, el diseño por bloques y la medición repetida del número de hojas enfermas sobre la misma planta en dos épocas diferentes:

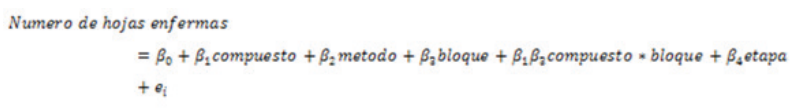

Numero de hojas enfermas

$+e_{i}$
Se evaluaron los supuestos del modelo y se identificó si existen diferencias con un nivel de significancia de 0,05 entre los métodos, entre los compuestos y el posible efecto de interacción entre el compuesto y el bloque.

Para la implementación de modelos lineales mixtos generalizado (GLMMs) se empleó el paquete glmmADMB, del software R. Este paquete tiene la capacidad de adicionar en el modelo la distribución de la variable respuesta de tipo Poisson. Además de permitir evaluar el efecto por mediciones repetidas y por el uso de bloques.

\section{Resultados y discusión}

La Roya del café comienza su infección por el envés de las hojas, aparece como una mancha que aumenta de tamaño y produce un polvo ligero color naranja. Dicho polvo son esporas del hongo las cuales necesitan de una mínima cantidad de agua, como un rocío de lluvia, para empezar a germinar. También requieren una temperatura entre $21-25^{\circ} \mathrm{C}$ y un período oscuro de por lo menos seis horas; es decir, solo requieren de una noche fría para comenzar su proceso destructivo en las plantas.

El proceso infectivo que ocurre de manera natural se replicó en este trabajo. Inicialmente, se realizó un tratamiento preventivo a un total de 36 plantas, que posteriormente fueron infectadas con la Roya. También se realizó un tratamiento curativo a 36 plantas que fueron infectadas con el hongo. En los tratamientos se evaluó la capacidad del compuesto aplicado de inhibir la formación del hongo. Esto se analizó mediante la medida de las hojas enfermas en cada planta (figura 2): 
Figura 2. Cantidad de plantas de café con el total de hojas con presencia de roya después de la aplicación de los quitosano con bajo grado de desacetilación (QBGD), quitosano con medio grado de desacetilación (QMGD), quitosano con bajo peso molecular (QBPM), quitosano con medio peso molecular (QMPM), solución de $\mathrm{HCl}$ 0,1 M (ACIDO) y testigo (AGUA).

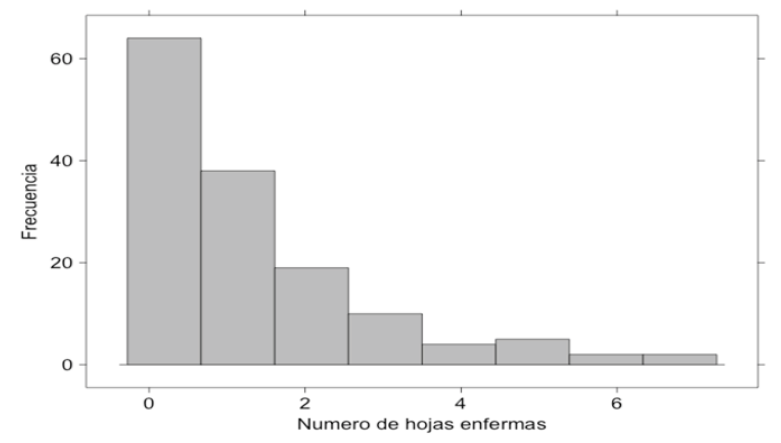

En la figura 2 se presenta la cantidad de hojas enfermas encontradas en relación a la cantidad de plantas de café. Después de aplicar los diferentes tratamientos a las 72 plantas evaluadas, se observa que aproximadamente 40 de ellas tuvieron una hoja enferma con Roya, al menos 20 de ellas tuvieron dos hojas enfermas y aquellas que presentaron entre 0 y 6 hojas enfermas estuvieron en una cantidad menor a 10 plantas. Este resultado indica que hubo una inhibición en la enfermedad. Se estima que si no se hubiera aplicado ningún compuesto lo más probable sería que todas las plantas presentaran una manifestación de la infección igual en cantidad de número de hojas enfermas, ya que todas se trataron de forma homogénea y se sometieron a las mismas condiciones de incubación.

No obstante, en las plantas de café que se cultivan en Colombia el manejo agronómico es vital para que la enfermedad del hongo no sea tan devastadora. Entre los manejos adecuados se encuentran: la erradicación de plantas tipo maleza que compiten por los nutrientes; una adecuada fertilización; poca sombra y una aplicación adecuada de los fungicidas. Para controlar este último factor se han diseñado estrategias de aplicación de los fungicidas en épocas determinadas del cultivo de café. Los fungicidas tienen efecto protector, como por ejemplo los compuestos cúpricos. Éstos se aplican antes de que aparezca la enfermedad. También se aplican otros productos que tienen efecto sistémico o curativo, cuando ya está instaurada la enfermedad (Rivillas Osorio et al., 2011). De acuerdo a esta práctica agrícola común, se evaluó la posible capacidad preventiva o curativa de los compuestos de quitosano como se describe en la metodología anterior, los resultados encontrados se observan en la figura 3.

Figura 3. Cantidad de plantas de café con hojas que tienen presencia de roya después de aplicar método preventivo (izquierda) y método curativo (derecha), con los compuestos quitosano bajo grado de desacetilación (QBGD), quitosano medio grado de desacetilación (QMGD), quitosano bajo peso molecular (QBPM), quitosano medio peso molecular (QMPM), solución de $\mathrm{HCl}$ 0,1 M (ACIDO) y testigo (agua).

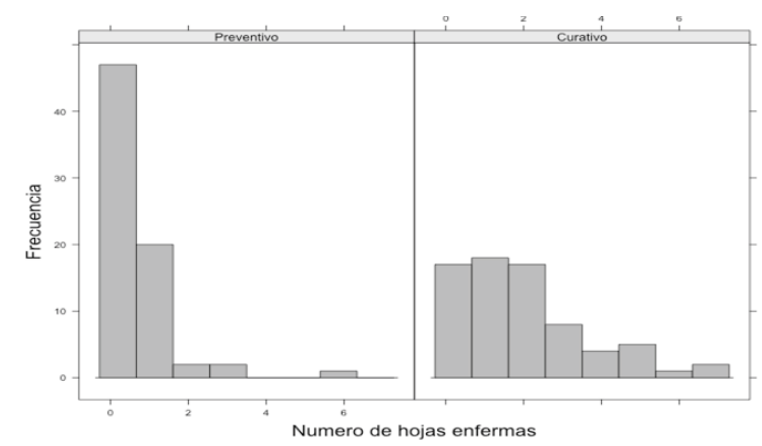

Al lado iquierdo se presentan los resultados con el metodo preventivo y al lado derecho los 
resultados con el método curativo. En el método preventivo se observa que aproximadamente 50 hojas no presentan señal de engermedad, al menos 20 plantas poseen una hoja enferma y menos de 10 plantas con más de tres hojas enfermas. En cambio en el metodo curativo los resultados muestran que la mayoría de las plantas tuvieron entre cero y seis hojas enfermas. Estos valores indican una efectividad del compuesto quitosano como tratamiento preventivo.

Con el fin de evidenciar el comportamiento de cada compuesto de quitosano (QMPM, QMGD, QBPM y QBGD) y observar si existían diferencias en la efectividad de éstos se representó en la figura 4 la cantidad de plantas vs el número de hojas enfermas para cada compuesto.

Figura 4. Cantidad de plantas con hojas enfermas según el compuesto aplicado quitosano bajo grado de desacetilación (QBGD), quitosano medio grado de desacetilación (QMGD), quitosano bajo peso molecular (QBPM), quitosano medio peso molecular (QMPM), solución de $\mathrm{HCl} \mathrm{0,1} \mathrm{M}$ (ACIDO) y testigo (AGUA).

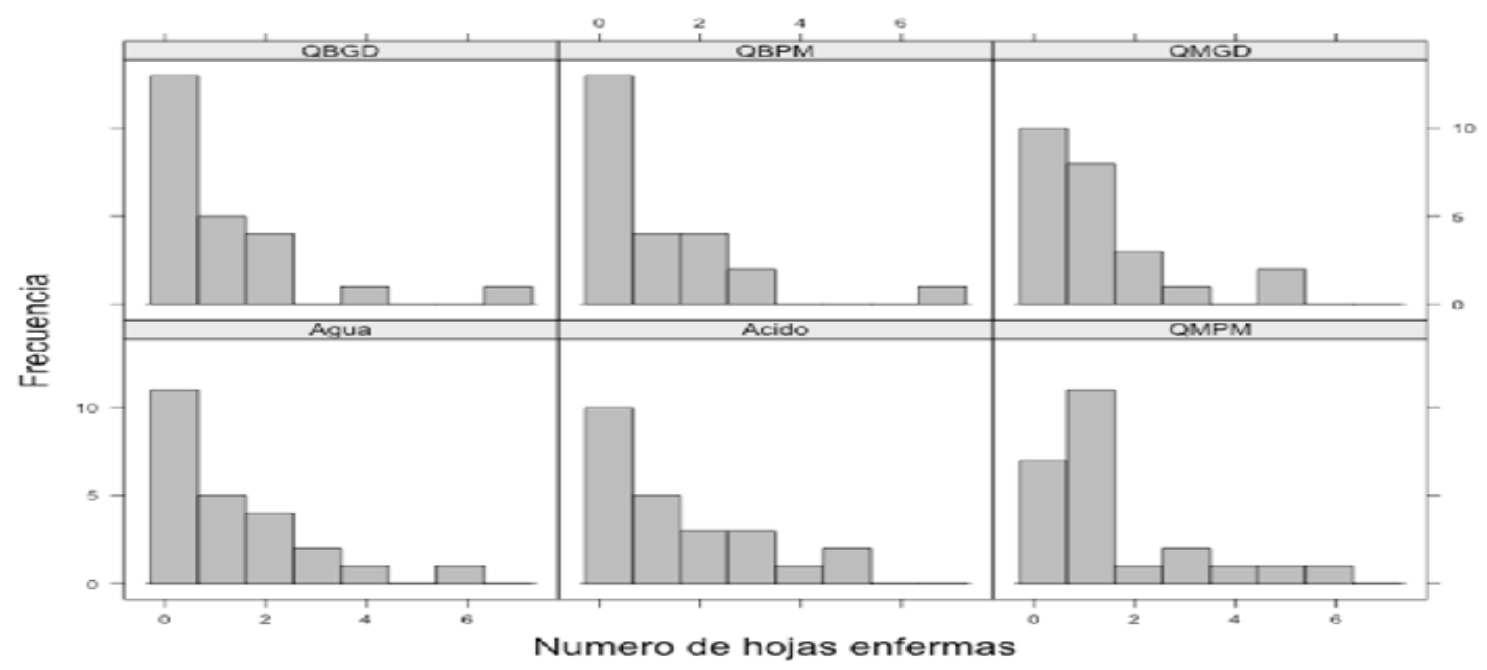

Según estos resultados la mayoría de los compuestos muestran un comportamiento similar entre sí. Excepto para los compuestos de quitosano de bajo grado de desacetilación (QBGD) y quitosano de bajo peso molecular (QBPM) en los que se observa una mayor cantidad de plantas con cero hojas enfermas. Podría afirmarse que un quitosano de menor peso molecular se encuentra más fragmentado y puede ser asimilado de manera más fácil por la planta, al igual que un quitosano sin tantos grupos acetilo que al tener menos impedimento en su estructura puede interactuar de forma más directa, propiedades que incrementan la efectividad. Sin embargo, se requieren estudios más profundos para el quitosano en estas dos formas.

Durante la realización del experimento se evaluó también la efectividad de acuerdo a la época de infección. Se estableció esta época como inicio de la enfermedad y final de la enfermedad; las cuales se determinaron comparando el avance de la infección durante seis semanas hasta que la roya alcanzó su fase máxima de infección en plantas que no tenían ningún tratamiento con aquellas que si tenían tratamiento. En la figura 5 se presenta la cantidad de plantas de café de 
seis meses a las de seis semanas después de la inoculación

Figura 5. Cantidad de plantas de café de seis meses a las de seis semanas después de la inoculación, con hojas con presencia de roya de acuerdo a la época de la infección: al inicio y al final, luego de la aplicación de quitosano bajo grado de desacetilación (QBGD), quitosano medio grado de desacetilación (QMGD), quitosano bajo peso molecular (QBPM), quitosano medio peso molecular (QMPM) y solución de $\mathrm{HCl}$ 0,1 M (ACIDO).

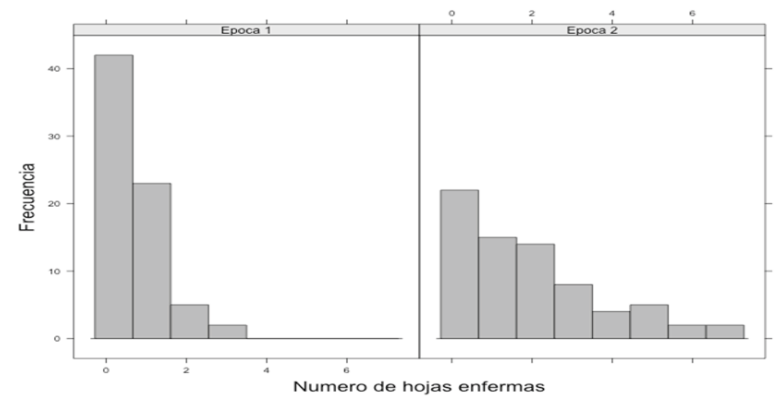

La época 1 corresponde al inicio de la infección y la época 2 al final de la infección. Como se observa, en la época 1, la cantidad de plantas con cero hojas enfermas fue mayor al inicio de la infección. Mientras que en la época 2, no se observan diferencias significativas que indiquen efectividad de los compuestos una vez las plantas presentaban la infección. Esto quiere decir que no hay efectividad de los compuestos una vez que la Roya se ha establecido en la planta. Por lo tanto, se puede sugerir un potencial para los compuestos evaluados como tratamiento fungicida tipo preventivo.

Por último, se necesitaba conocer si había algún impacto en la aplicación de bloques al azar que modificara la efectividad de los compuestos. En la figura 6 se presenta el número de hojas enfermas por bloque. Existió mayor frecuencia de conteo de ceros de hojas enfermas en los bloques 3 y 5 es decir menos hojas infectadas en los bloques.

Figura 6. Histograma del número de hojas de plantas de café de seis meses de edad con presencia de roya en cada bloque de almácigos, luego de la adición de quitosano bajo grado de desacetilación (QBGD), quitosano medio grado de desacetilación (QMGD), quitosano con bajo peso molecular (QBPM), quitosano medio peso molecular (QMPM), solución de $\mathrm{HCl}$ 0,1 M (ACIDO) y testigo (AGUA).

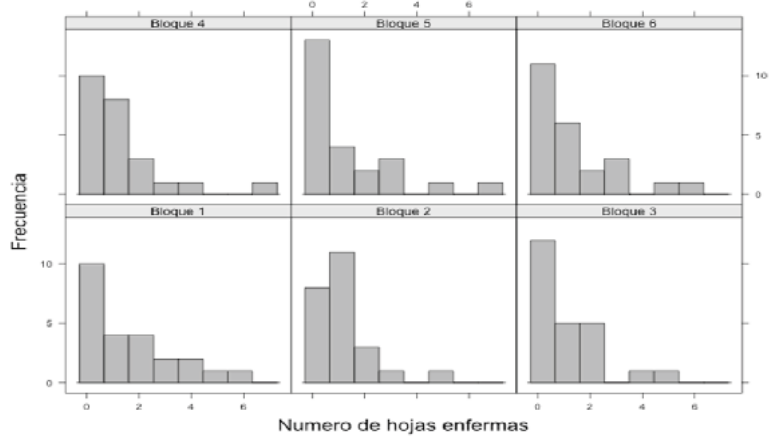

En general, se observó un exceso de conteo de ceros, lo cual es favorable porque hay poca cantidad de hojas infectadas. Para comprobar todo el comportamiento observado anteriormente, se aplicó un modelo de regresión mixto binomial negativo con ceros inflados para evaluar el análisis de efectividad del quitosano sobre la roya. El modelo incluyó las variables que mostraron tener indicios de efectividad como método, época, compuesto y bloque.

Al ajustar el modelo de regresión de Poisson se encontró que los factores método, época e interacción del compuesto por bloque son estadísticamente significativos $(\alpha 0,05)$. Se incorporó al modelo estadístico las mediciones de la época, que corresponden a mediciones repetidas sobre la misma planta, encontrando el modelo mixto binomial negativo con ceros 
inflados. Posteriormente, con el análisis del modelo se encontraron diferencias estadísticamente significativas entre el número de hojas enfermas promedio de los métodos y la época.

Dentro del bloque 5 se encontraron diferencias entre el agua y QBPMy QBGD, respectivamente. Mientras que en el bloque 6 se encontraron diferencias entre el número de hojas enfermas promedio al aplicar el agua, QMPM y QMGD, respectivamente (tabla 1). Esto quiere decir que al haber diferencias estadísticas para estos bloques se evidencia que hay efectividad entre los compuestos mencionados pues al haber efectuado una aleatorización por bloques se elimina la variabilidad estadística debida a las unidades experimentales y se deduce que dicha variabilidad encontrada es debida a los compuestos evaluados.

Estudios realizados por otros investigadores indican que el poder fungicida del quitosano influye directamente sobre los hongos afectando la germinación, esporulación y aumento del micelio. Dicha acción fungicida se debe a la interacción de los grupos amino que en medio ácido se cargan de forma positiva, con los residuos de macromoléculas en la pared de los hongos, lo cual modifica la permeabilidad de la membrana plasmática y altera las funciones normales del hongo; también se ha encontrado que altera las células del patógeno causando deficiencia en su formación (Guerra-Sánchez, Vega-Pérez, Velázquez-del Valle \& Hernández-Lauzardo, 2008; Lárez Velasquez, 2008; Sánchez-Domínguez, Bautista-Baños \& Castillo Ocampo, 2007; Yien, Zin, Sarwar \& Katas, 2012; Ziani, Fernández-Pan, Royo \& Mate, 2009).

Se pudo observar en esta investigación que hubo diferencias entre los métodos preventivo y curativo. El número de hojas infectadas en total fue menor en el primero, lo cual muestra la efectividad del quitosano para detener el progreso de la infección. Con esto se evidencia que el quitosano en general presenta mayor efectividad como método preventivo. La razón de este comportamiento puede deberse a que el quitosano forma una película semipermeable sobre la superficie aplicada (Hernández-Lauzardo,Hernández-Martínez \& Velázquez-del Valle, 2007). Es probable que dicho recubrimiento protegiera a la planta de café del ataque de roya.

Las plantas a las que se les aplicaron los compuestos no mostraron daños posteriores a la aplicación como marchitamiento o muerte. También dicha respuesta puede obedecer al efecto inductor que tiene el quitosano, este compuesto reduce la maceración celular que produce un hongo patógeno y estimula la producción de hidrolasas antifúngicas (quitinasas y $\beta$ 1-3 glucanasas) las cuales actúan sobre la pared celular del patógeno disminuyendo su crecimiento. Además, induce a la formación de barreras estructurales al formar compuestos fenólicos que produce la enzima fenil alanina amonio liasa, la cual se incrementa con la presencia de quitosano; estos fenoles formados limitan el avance del patógeno; otro compuesto que forma una barrera estructural cuando se aplica el quitosano es la lignina que aumenta en presencia del compuesto evaluado (Hernández-Lauzardo et al, 2007; Rodríguez-Pedroso et al, 2009). En otras palabras, el quitosano hace que la planta incremente sus defensas lo que la hace más fuerte ante el ataque de patógenos.

En los resultados del modelo de regresión (tabla 1) se observaron las diferencias entre bloques y compuestos. Estas diferencias están en el bloque 5 entre QBPM, QBGD y el testigo (agua); y en el bloque 6 entre QMPM y QMGD 
y el testigo (agua). Los resultados estimados no permiten conocer cuál de las formas del quitosano es más efectiva y por qué se observaron dichas diferencias en estos bloques y no en los otros; ya que es necesario controlar el efecto de los factores perturbadores como el viento, la humedad relativa y la temperatura que pudieron influir en los resultados. No obstante, las diferencias encontradas indican que es posible que algunas formas de quitosano sean más efectivas que otras, como la del quitosano de bajo peso molecular el cual ha mostrado inhibición del crecimiento micelial en Rhizopus Stolonifer (Hernández-Lauzardo et al., 2008), Botrytis cinérea (Badawy \& Rabea, 2009), etc., pero requiere de estudios posteriores e independientes por compuesto a evaluar.

\section{Conclusiones}

El quitosano evaluado en sus diferentes formas mostró una efectividad contra la roya cuando se aplicó como método preventivo. Posiblemente porque las características del compuesto le permiten formar una película sobre las hojas que evita el ingreso del hongo; dichos resultados se confirman al mostrar efectividad al inicio de la infección reforzando así la afirmación de que su poder fungicida se encuentra en su capacidad de proteger a la planta.

No obstante, también se encontró una efectividad en las formas de quitosano con bajo peso molecular y bajo grado de desacetilación que indican que entre menor es su tamaño y su impedimento estérico, mayor puede ser su efectividad. Estas formas del compuesto merecen ser estudiadas más a fondo en otros trabajos de investigación. Al ser el quitosano efectivo contra la roya se convierte en un compuesto capaz de combatir el hongo y que no es tóxico como los fungicidas actuales aportando dos grandes beneficios: el aprovechamiento de un subproducto como el exoesqueleto de camarón y el beneficio a la fauna y flora por ser biodegradable en el medio ambiente. Además, este subproducto se encuentra en abundancia en el Valle del Cauca, lo cual podría impulsar el desarrollo de una nueva industria y otras fuentes de empleo para la región.

\section{Agradecimientos}

Los autores agradecen a Yenifer Orobio por su gran ayuda en la aclaración de dudas estadísticas presentadas durante la realización de esta investigación. También a Ghisliane Echeverry Prieto por todas sus sugerencias y aportes con relación a la parte química.

\section{Referencias bibliográficas}

Aider, M. (2010). Chitosan application for active bio-based films production and potential in the food industry: Review. LWT - Food Science and Technology, 43(6), 837-842. doi: http://dx.doi. org/10.1016/j.lwt.2010.01.021

Badawy, M. E. I, \& Rabea, E. I. (2009). Potential of the biopolymer chitosan with different molecular weights to control postharvest gray mold of tomato fruit. Postharvest Biology and Technology, 51(1), 110-117. doi: http://dx.doi. org/10.1016/j.postharvbio.2008.05.018

Cristancho, M., Rozo, Y, Escobar, C, Rivillas, C \& Gaitán, Á. (2012). Razas de roya: epidemias de 2008-2011. Avances Técnicos Cenicafé, 2012(1), 1-8. 
Guerra-Sánchez, M. G, Vega-Pérez, J, Lárez Velasquez, C. (2008). Algunas Velázquez-del Valle, M. G., \& Hernández-Lauzardo, A. N. (2008). Antifungal activity and release of compounds on Rhizopus stolonifer (Ehrenb.:Fr.) Vuill. by effect of chitosan with different molecular weights. Pesticide Biochemistry Physiology 93(1), 18-22. doi: http://dx.doi. org/10.1016/j.pestbp.2008.09.001

Hadwiger, L. A. (2013). Multiple effects of chitosan on plant systems: Solid science or hype. Plant Science, 208, 42-49. doi: http://dx.doi.org/10.1016/j. plantsci.2013.03.007

Hernández-Lauzardo, A. N., Bautista-Baños, S., Velázquez-delValle,M.G.,Méndez-Montealvo, M. G., Sánchez-Rivera, M. M., \& Bello-Pérez, L. A. (2008). Antifungal effects of chitosan with different molecular weights on in vitro development of Rhizopus stolonifer (Ehrenb.:Fr.) Vuill. Carbohydrate Polymers, 73(4), 541-547. doi: http://dx.doi.org/10.1016/j. carbpol.2007.12.020

Hernández-Lauzardo, A. N, Hernández-Martínez, M, \& Velázquez-del Valle, M. G. (2007). Actividad Antifúngica del Quitosano en el ControldeRhizopus stolonifer(Ehrenb.:Fr.) Vuill. y Mucor spp. Revista Mexicana de Fitopatología, 25(1), 109-113.

Kean, T., \& Thanou, M. (2010). Biodegradation, biodistribution and toxicity of chitosan. Advanced Drug Delivery Reviews, 62(1), 3-11. doi: http://dx.doi.org/10.1016/j. addr.2009.09.004

potencialidades de la quitina $y$ el quitosano para usos relacionados con la agricultura en Latinoamérica. Revista UDO Agrícola, 8(1), 1-22.

Montes, C., Armando P, O., \& Cadena, R. (2012). Infestación e incidencia de broca, roya y mancha de hierro en cultivo de café del Departamento del Valle del Cauca. Biotecnología en el Sector Agropecuario y Agroindustrial, 10(1), 98-108.

Prapagdee, B., Kotchadat, K., Kumsopa, A., \& Visarathanonth, N. (2007). The role of chitosan in protection of soybean from sudden death syndrome caused by Fusarium solani f. sp. glycines. Bioresource Technology, 98(1), 1353-1358. doi: http://dx.doi. org/10.1016/j.biortech.2006.05.029

Rivillas Osorio, C., Serna Giraldo, C., Cristancho Ardila, M., \& Gaitán Bustamante, A. L. (2011). La Roya del Cafeto en Colombia: Impacto, manejo y costos de control. Boletin Técnico Cenicafé, 2011, 1-53.

Rodríguez-Pedroso, A. T., Ramírez-Arrebato, M. A., Rivero-González, D., Bosquez-Molina, L. E., Barrera-Necha, L., \& Bautista-Baños, S. (2009). Propiedades Químico Estructurales y actividad biológica de la quitosana en microorganismos fitopátogenos. Revista Chapingo, serie horticultura, 15(3), 307-317. 
Rodriguez, A., Ramírez, M., Cárdenas, R. Ziani, K., Fernández-Pan, I., Royo, M., \& Mate, M., Rodriguez, A., \& Bautista Baños, S. (2006). Efecto de la quitosana en la inducción de la actividad de enzimas relacionadas con la defensa y protección de Plántulas de arroz (Oryza sativa L.) contra Pycularia grisea Sacc. Revista Mexicana de Fitopatología, 24(1), 1-7.

Sánchez-Domínguez, D., Bautista-Baños, S., \& Castillo Ocampo, P. (2007). Efecto del quitosano en el desarrollo y morfología de Alternaria alternata ( $\mathrm{Fr}$.) Keissl. Anales de Biología, 29, 23-32.

Van, S. N., Minh, H. D., \& Anh, D. N. (2013). Study on chitosan nanoparticles on biophysical characteristics and growth of Robusta coffee in greenhouse. Biocatalysis and Agricultural Biotechnology, 2(4), 289-294. doi: http://dx.doi.org/10.1016/j. bcab.2013.06.001

Yien, L., Zin, N. M., Sarwar, A., \& Katas, H. (2012). Antifungal Activity of Chitosan Nanoparticles and Correlation with Their Physical Properties. International Journal of Biomaterials, 2012, 1-9. doi: http://dx.doi.org/10.1155/2012/632698

Younes, I., Sellimi, S., Rinaudo, M., Jellouli, K., \& Nasri, M. (2014). Influence of acetylation degree and molecular weight of homogeneous chitosans on antibacterial and antifungal activities. International Journal of Food Microbiology, 185, 57-63. doi: http://dx.doi.org/10.1016/j. ijfoodmicro.2014.04.029 